\title{
Surface Study of Nb/Cu Films for Cavity Deposition by ECR Plasma*
}

\author{
A.T. $\mathrm{Wu}^{\#}$, R. Ike, H.L. Phillips, A.-M. Valente, H. Wang, and G. Wu \\ Thomas Jefferson National Accelerator Facility, Newport News, VA 23606, USA
}

\begin{abstract}
Niobium $(\mathrm{Nb})$ thin film deposited on copper $(\mathrm{Cu})$ cavities through electron cyclotron resonance (ECR) plasma appears to be an attractive alternative technique for fabricating superconducting radio frequency cavities to be used in particle accelerators. The performance of these obtained $\mathrm{Nb} / \mathrm{Cu}$ cavities is expected to depend on the surface characteristics of the $\mathrm{Nb}$ films. In this report, we investigate the influence of deposition energy on surface morphology, microstructure, and chemical composition of $\mathrm{Nb}$ films deposited on small $\mathrm{Cu}$ disks employing a metallographic optical microscope, a 3-D profilometer, a scanning electron microscope, and a dynamic secondary ion mass spectrometry. The results will be compared with those obtained on $\mathrm{Nb}$ surfaces treated by BCP, EP, and BEP.
\end{abstract}

\section{INTRODUCTION}

Niobium $(\mathrm{Nb})$ coating on copper $(\mathrm{Cu})$ cavities is an attractive technique for fabricating superconducting radio frequency cavities in comparison with other techniques based on bulk $\mathrm{Nb}$ for the application in particle accelerators, due to a significant material cost reduction and a better thermal stability at temperatures near $4.2 \mathrm{~K}$. However, the commonly used cylindrical magnetron sputtering technique for depositing $\mathrm{Nb}$ film as developed by CERN for LEP2 suffers from a rapid increase of residual resistance [1] at accelerating gradients above 15 $\mathrm{MV} / \mathrm{m}$ at $1.7 \mathrm{~K}$, leading to the degradation in the quality factor $\mathrm{Q}$ of the coated $\mathrm{Cu}$ cavities. One of the major drawbacks of cylindrical magnetron sputtering technique is that the arrival angle of $\mathrm{Nb}$ flux on the inner surface of $\mathrm{a} \mathrm{Cu}$ cavity is not constant. The angle varies from beam pipe to equator. In some cases [2], the angle variation can be continuously from $0^{\circ}$ to $90^{\circ}$. Simulation using 3-D film growth code SIMBAD [3] and real experiment [4] have demonstrated that films grow at oblique incident angles are highly porous and the porosity can be as high as $80 \%$ [4] due to the well-known shadowing effect. Besides the adatom mobility of this technique is limited and the distance between cathode and anode is variable, porous columnar growth of $\mathrm{Nb}$ is inevitable in some areas on the inner surface of a $\mathrm{Cu}$ cavity. Therefore surface of the $\mathrm{Nb}$ film can be very rough that may result in an increase in surface residual resistance [5]. Most importantly, perhaps, is that porous columnar microstructure may provide an easy channel for oxygen to penetrate into $\mathrm{Nb}$ film, leading to the formation of some sub-oxides that may not be superconductors or may be superconductors at lower temperatures than that of $\mathrm{Nb}$ and causing RF losses. These drawbacks can, in principal, be overcome by an energetic thin film

\footnotetext{
"Supported by DOE Contract DE-AC05-84ER40150.

\# andywu@jlab.org
}

deposition technique [6] through generating $\mathrm{Nb}$ plasma via the electron cyclotron resonance method $[7,8]$.

In this paper, we report our preliminary results on surface characterization on $\mathrm{Nb}$ films deposited on flat $\mathrm{Cu}$ coupons using the energetic deposition technique [6]. The aim is to study how the variations of deposition energy can affect morphology and surface oxide layer structure of $\mathrm{Nb}$ films, in preparation for actual deposition on a 500 $\mathrm{MHz}$ single cell elliptical $\mathrm{Cu}$ cavity. Due to the limited space, results are briefly presented.

\section{EXPERIMENT}

Four samples were used in this study. They were electro polished $\mathrm{Cu}$ disks of 2.5 " diameter and coated with $\mathrm{Nb}$ films using the energetic deposition technique [6]. Deposition was done at a base pressure of $3 \times 10^{-6}$ (Pa). About $150 \mu \mathrm{m}$ was removed by electro polishing $\left(55 \% \mathrm{H}_{3} \mathrm{PO}_{4}\right.$ and $\left.45 \% \mathrm{C}_{4} \mathrm{H}_{10} \mathrm{O}\right)$ from each $\mathrm{Cu}$ disk. Deposition energy was controlled by biasing the $\mathrm{Cu}$ disks with voltages of $60,70,80$, and $90 \mathrm{~V}$ respectively (for simplicity, the corresponding samples are denoted as $60 \mathrm{Cu}, 70 \mathrm{Cu}, 80 \mathrm{Cu}$, and $90 \mathrm{Cu}$ ). A retarding field energy analyzer mounted on the substrate without bias voltage showed that arriving $\mathrm{Nb}$ ions had a kinetic energy of $63 \mathrm{~V}$ with energy spread of $20 \mathrm{eV}$ full width at half maximum.

Optical measurements were done using a computer controlled metallographic optical microscope (MOM) made by Carl Zeiss. An Amray 1830 scanning electron microscope (SEM) was using for surface observation at larger magnifications. Surface roughness of the samples was quantitatively evaluated by a KLA-Tencor P-15 profilometer. This profilometer can do 3-D scans over an area as large as $80 \mathrm{X} 200 \mathrm{~mm}^{2}$ with a guaranteed vertical repeatability of $0.75 \mathrm{~nm}$. Surface chemical information and depth profile were measured via a home-made dynamic secondary ion mass spectrometer (SIMS). For the details about the characterization devices used in this study, please see reference 9 .

\section{RESULT AND DISCUSSION}

A typical sample is shown in Fig.1. Arrows in Fig.1 indicate $\mathrm{Cu}$ surface that is used for MOM observations to check the smoothness of the $\mathrm{Cu}$ substrate. Generally speaking, all surfaces of the electro polished $\mathrm{Cu}$ disks look similar under MOM. Observations at larger magnifications reveal that the $\mathrm{Cu}$ surfaces of $50 \mathrm{Cu}$ and $90 \mathrm{Cu}$ are a little smoother than those of the other two. Quantitative information regarding the smoothness of the deposited $\mathrm{Nb}$ surfaces is obtained through 3-D scans on the surfaces using the P-15 profilometer. Surface roughness is found to vary dramatically from one area to the other. Scans over areas smaller than $4000 \times 4000$ 


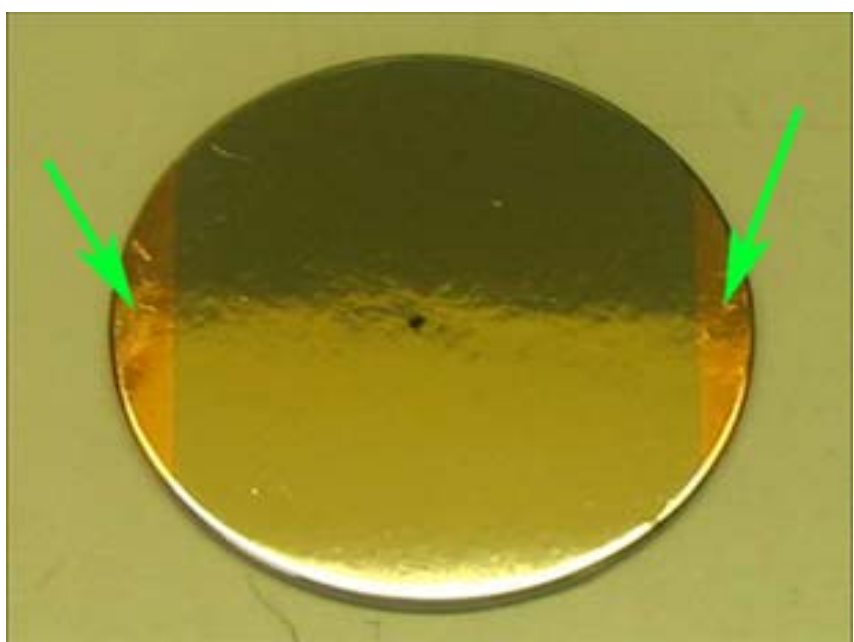

Fig.1: A typical sample.

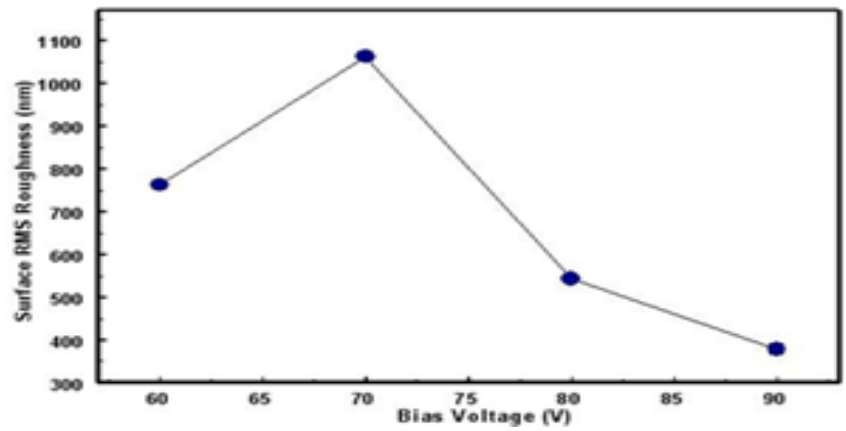

Fig.2: Average surface RMS roughness Vs the bias voltage extracted from 3-D profilometer scans over areas of $4000 \times 4000 \mu^{2}$ on $\mathrm{Nb} / \mathrm{Cu}$ samples (see the text for details).
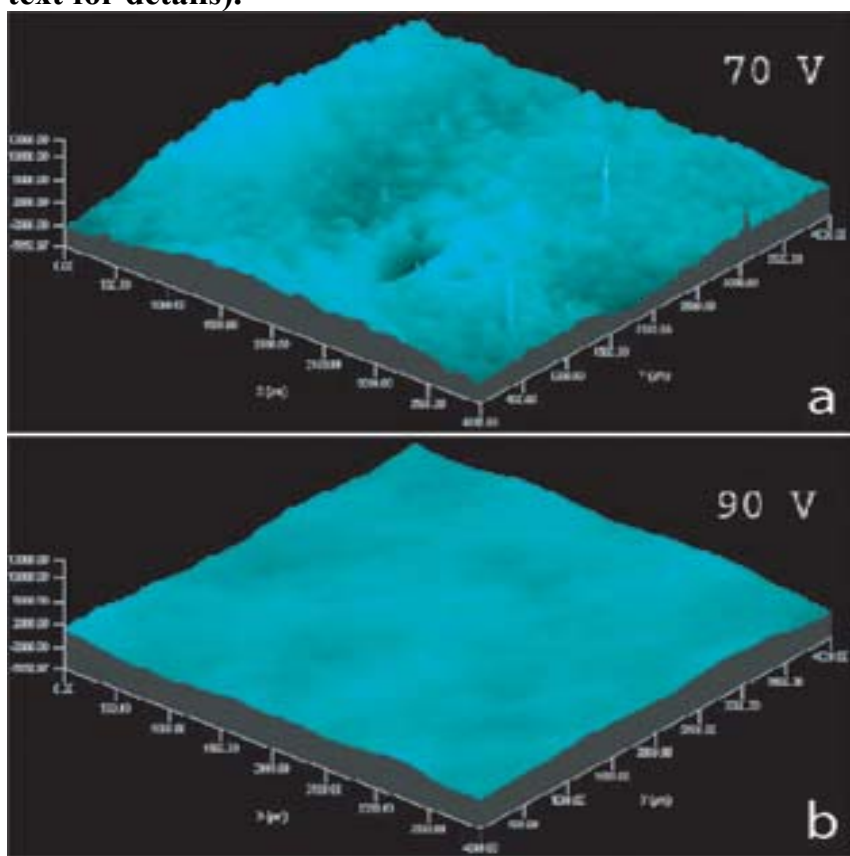

Fig.3: Typical profilometer images of $\mathrm{Nb}$ films deposited at bias voltages of a) $70 \mathrm{~V}$ and b) $90 \mathrm{~V}$.

$\mu \mathrm{m}^{2}$ show a standard deviation larger than $10 \%$. Fig. 2 summarizes the values of average root mean square
(RMS) extracted from profilometer measurements. Each RMS value shown in Fig.2 is an average of five scans over areas of $4000 \mathrm{X} 4000 \mu \mathrm{m}^{2}$ randomly selected on the surface of a sample. Typical profilometer images for $70 \mathrm{Cu}$ and $90 \mathrm{Cu}$ are shown in Fig. 3

It appears that the large standard deviation for scans smaller than $4000 \times 4000 \mu \mathrm{m}^{2}$ is mainly a result of a relatively large smoothness variation on the $\mathrm{Cu}$ substrates as shown in an extreme case in Fig.4. On the other hand, it is difficult to attribute the variation in Fig.2 to the smoothness variation in substrates alone. For instance, the RMS roughness of $60 \mathrm{Cu}$ is almost twice as much as that of $90 \mathrm{Cu}$. But the $\mathrm{Cu}$ surface of $60 \mathrm{Cu}$ looks almost the same or even a little smoother than that of $90 \mathrm{Cu}$ under MOM. Therefore, our results seem to show that a higher deposition energy does tend to result in a smoother $\mathrm{Nb}$ surface due to a higher adatom mobility. SEM measurements do not give any additional meaningful information due to its deep depth of field.

A comparison of surface RMS roughness of $90 \mathrm{Cu}$ with a typical $\mathrm{BCP}, \mathrm{EP}, \mathrm{BEP}$, and $\mathrm{Nb}$ single crystal samples is given in Table 1.

Table 1: RMS roughness extracted from 3-D profilimeter scans over areas of $200 \times 200 \mu^{2}$ for typical $90 \mathrm{Cu}, \mathrm{Nb}$ single crystal (NbSC), Nb surfaces treated by BCP, EP, and buffered EP (BEP).

\begin{tabular}{|c|c|c|c|c|c|}
\hline $\begin{array}{c}\text { Preparation } \\
\text { Method }\end{array}$ & BCP & EP & $\begin{array}{c}\text { BEP } \\
{[10]}\end{array}$ & $\begin{array}{c}\text { NbSC } \\
{[11]}\end{array}$ & 90Cu \\
\hline RMS (nm) & 1274 & 251 & 35 & 27 & 94 \\
\hline
\end{tabular}

SIMS measurements are done at a base pressure of 9.5 $\times 10^{-9}$ Torr with an Ar primary ion beam at an ion beam energy of $2.7 \mathrm{KeV}$. A typical SIMS spectrum from 1 to 300 AMU obtained on $90 \mathrm{Cu}$ is shown in Fig.5. Fig.6 is a typical SIMS spectrum on a Nb surface treated by the conventional 112 BCP. From the comparison between Figs. 5 and 6, we can see that the cracking pattern of surface oxide layer of the $\mathrm{Nb}$ film is different from that of $\mathrm{BCP}$ treated $\mathrm{Nb}$ surface as judging from the ratio between the intensities of $\mathrm{Nb}^{+}, \mathrm{NbO}^{+}$, and $\mathrm{NbO}_{2}^{+}$peaks, implying

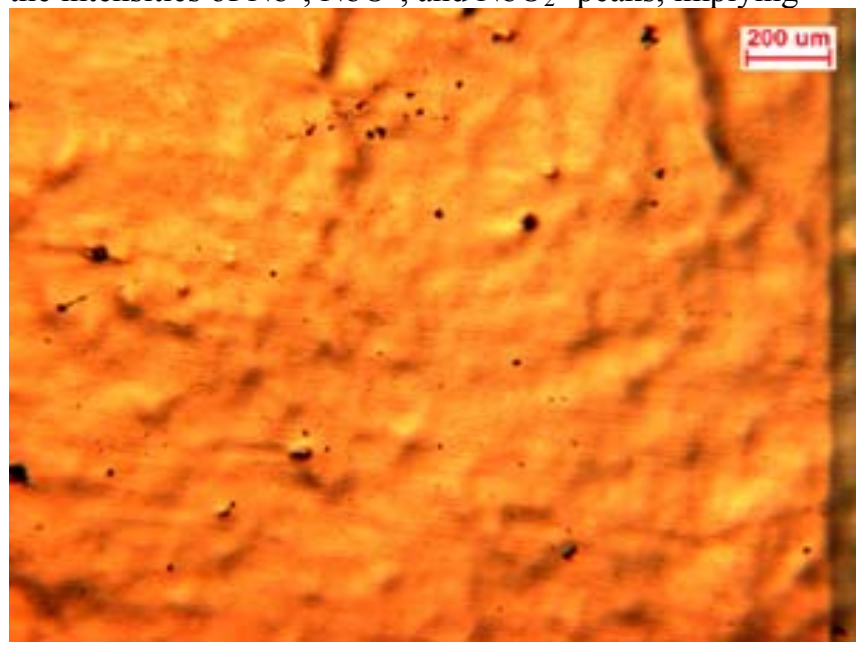

Fig.4: MOM image of the Cu surface of the $\mathrm{Nb}$ film deposited at a bias voltage of $70 \mathrm{~V}$. 
therefore that surface oxide layer structure may not be the same. The surface of $\mathrm{Nb}$ film also appears to be much less contaminated. The same observations are found on all other $\mathrm{Nb} / \mathrm{Cu}$ samples. Hydrogen may not be the major concern on our $\mathrm{Nb}$ films. SIMS depth profile measurements reveal that oxygen content is close or more in the interior of the films as compared with those of BCP and EP, except 90Cu (see Fig.7). If oxygen is the main impurity of the $\mathrm{Nb}$ films as implied from our SIMS data, this result should indicate that the superconducting transition width should be the narrowest for $90 \mathrm{Cu}$, which is not what observed [6]. Therefore, other small amounts of impurities may play some roles here and have to be measured by SIMS depth profiling too, if microstructures are similar. More SIMS measurements are underway. Detailed SIMS results will be published elsewhere.

\section{SUMMARY}

Preliminary results of surface characterization on $\mathrm{Nb}$ films deposited on 2.5" electro polished $\mathrm{Cu}$ disks under four different deposition energies of 123, 133, 143, and $153 \mathrm{eV}$ are reported. Measurements show that higher deposition energy can lead to a smoother $\mathrm{Nb}$ surface in the energy window studied. More $\mathrm{Nb}$ films should be made at deposition energy higher than $153 \mathrm{eV}$ to find the optimal energy that may give us the smoothest surface. $\mathrm{The} \mathrm{Nb}$ film surface deposited at a bias voltage of $90 \mathrm{~V}$ is smoother than typical $\mathrm{Nb}$ surfaces treated by the conventional BCP and EP techniques. The surfaces of $\mathrm{Nb}$ films have much less contaminations compared with those of BCP treated $\mathrm{Nb}$. SIMS measurements also reveal that surface oxide layer structure of the $\mathrm{Nb}$ films may not be the same as that of $\mathrm{BCP}$ treated $\mathrm{Nb}$ bulk samples and oxygen content is higher for some $\mathrm{Nb}$ films in the interior

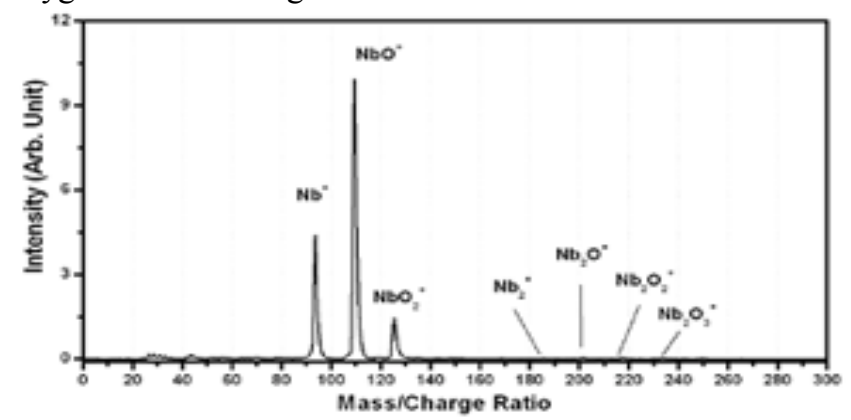

Fig.5: Typical SIMS spectrum on 90Cu surface.

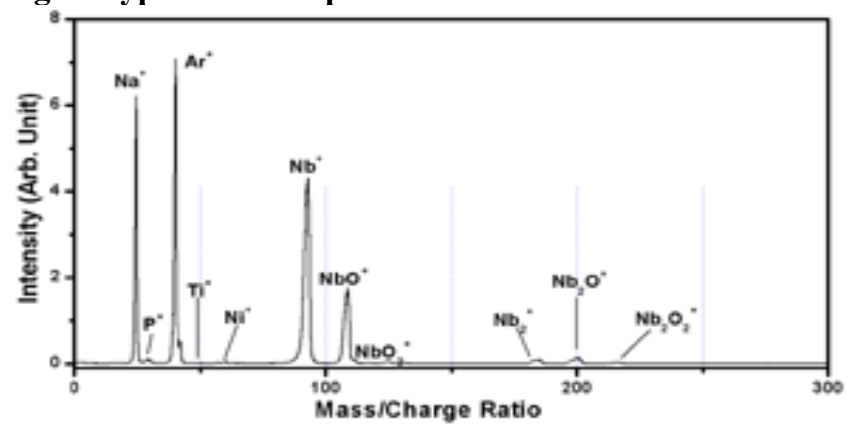

Fig.6: Typical SIMS spectrum on BCP treated Nb surface.
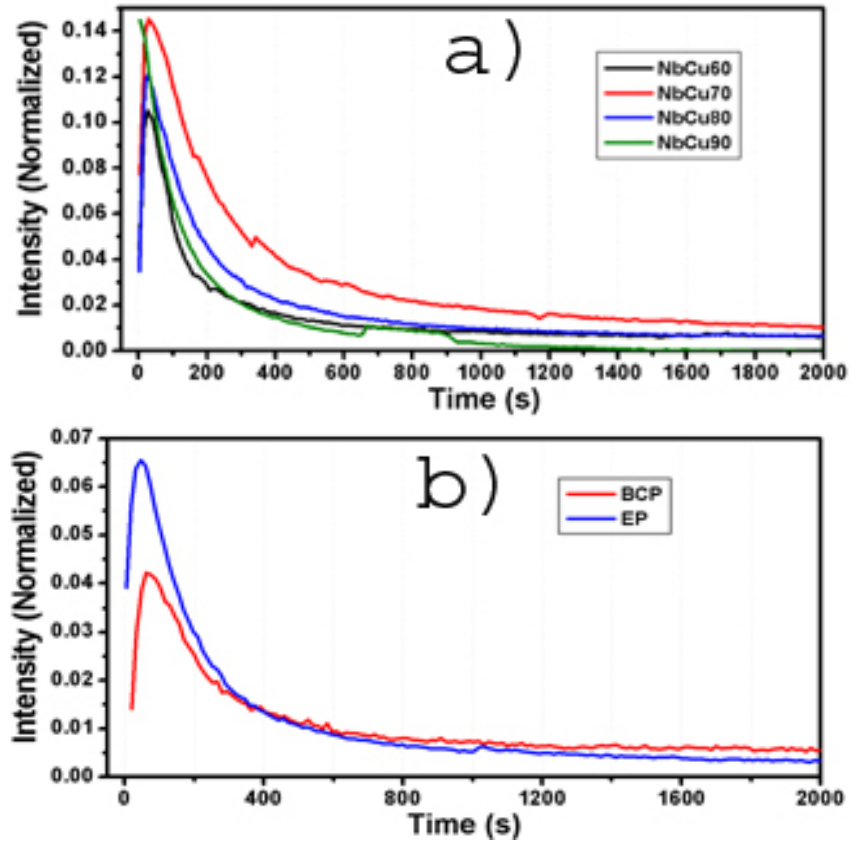

Fig.7: Typical SIMS oxygen depth profile plots for a) $60 \mathrm{Cu}, 70 \mathrm{Cu}, 80 \mathrm{Cu}$, and $90 \mathrm{Cu}, \mathrm{b}) \mathrm{BCP}$ and EP

of the films. A better base pressure may be necessary for the energetic deposition system in order to reduce oxygen content of the films. RF measurements are needed for comparison with the results from surface characterization.

\section{REFERENCES}

[1] C. Benvenuti, S. Calatroni, M. Hakovirta, H. Neupert, M. Prada, A.-M. Valente, Proc. of the $10^{\text {th }}$ Workshop on RF Superconductivity, Tsukuba, Japan, 2001, P252

[2] D. Tonini, C. Greggio, G. Keppel, F. Laviano, M. Musiani, G. Torzo, and V. Palmieri, Proc. of the $11^{\text {th }}$ Workshop on RF Superconductivity, Lubeck, Germany, 2003, ThP11

[3] T. Sym, D. Vick, M.J. Brett, S.K. Dew, A.T. Wu, J. Sit, and K. Harris, Journal of Vacuum Science \& Technology A, 18 (2000) P2507

[4] A.T. Wu and M.J. Brett, Sensors and Materials, 13 (2001) P399

[5] C. Benvenuti, S. Calatroni, M. Hakovirta, H. Neupert, M. Prada, A.-M. Valente, C.A. Van't Hoff, Physica C 351 (2001) P421

[6] G. Wu, L. Phillips, and R. Sundelin, Journal of Vacuum Science \& Technology A, 21 (2003) P842

[7] W.M. Holber et al, Journal of Vacuum Science \& Technology A, 11 (1993) P2903

[8] S.M. Rossnagel et al, Journal of Vacuum Science \& Technology B, 12 (1994) P449

[9] A.T. Wu, Proc. of the $11^{\text {th }}$ Workshop on RF Superconductivity, Lubeck, Germany, 2003, ThP13

[10] A.T. Wu, J. Mammossor, L. Phillips, J. Delayen, C. Reece, A. Wilkerson, D. Smith, and R. Ike, to be published

[11] G.R. Myneni et al, to be published 